\title{
Il braccio falso di Antonello Petrucci nella quarta novella del Novellino di Masuccio
}

\author{
Vincenzo VITALE \\ Università di Basilea
}

\begin{abstract}
Partendo dal presupposto del rapporto organico esistente tra le dediche e le novelle del Novellino di Masuccio Salernitano, il saggio propone un'analisi della quarta novella, dedicata ad Antonello Petrucci, umanista di umili natali diventato uno degli uomini più potenti del Regno di Napoli nella seconda metà del Quattrocento. Le sottili allusioni al dedicatario iscritte nella novella inducono a riconoscere un'accusa di baratteria negli apparenti elogi della dedica.
\end{abstract}

Keywords: Masuccio Salernitano, dedica, reliquie, Novellino, Napoli

Le dediche del Novellino di Tommaso Guardati detto Masuccio Salernitano sono legate alle rispettive novelle da un rapporto strettissimo, organico e originario. ${ }^{I}$ Nel Novellino il nesso tra novella e dedicatario è molto più sistematico rispetto al semplice grado di pertinenza richiesto dalla ricerca topica della convenienza, in virtù della quale la persona cui è offerta un'opera è scelta sulla base di affinità del testo con i suoi interessi, di rapporti personali eventualmente legati alla composizione dell'opera, di consonanze del contenuto con le sue convinzioni personali. ${ }^{2} \mathrm{Al}$ Novellino non è lecito applicare il naturale e automatico assunto secondo cui l'opera, una volta compiuta, è offerta come un organismo autonomo e conchiuso in se stesso al personaggio storico più confacente. Si può affermare viceversa che in Masuccio la persona del dedicatario sia logicamente anteriore al testo propriamente narrativo. La novella appare come un vestito cucito sulla misura specifica e individuale del ricevente. Il meccanismo del racconto è congegnato sulla scorta di un parallelismo sistematico con la vicenda biografica del dedica-

I Per l'indicazione e lo studio dei rapporti di implicazione sistematica tra le dediche e le novelle del Novellino mi permetto di rinviare a Vincenzo Vitale, La dedica ad Ariete: implicazioni anti-aragonesi nel 'Novellino' di Masuccio, in «Margini. Giornale della dedica e altro», 9, 2015, 24 pagine (http://www.margini.unibas.ch/web/it/index.html) e a Id., Dottor Pontano e fra Pontano nella terza novella del Novellino di Masuccio, in «Studi e problemi di critica testuale», XCIV, I, 20I7, pp. 2I-49.

2 Per l'esplicitazione topica delle ragioni della scelta del dedicatario nella prassi dedicatoria fino al Settecento cfr. Maria Antonietta Terzoli, I testi di dedica tra secondo Settecento e primo Ottocento: metamorfosi di un genere, in Dénouement des Lumières et invention romantique, Actes $d u$ Colloque de Genève, 24-25 novembre 2000, Textes réunis par Giovanni Bardazzi et Alain Grosrichard, Genève, Droz, 2003, pp. I6I-192, in partic. I66-I67; per la regola della convenienza cfr. Marco Paoli, La dedica: storia di una strategia editoriale (Italia, secoli XVI-XIX), Lucca, Maria Pacini Fazzi, 2009, pp. 24-25 e 6o-64. 
tario, rispecchiata in modo allusivo e diffratto nel corpo della novella. $\mathrm{Si}$ tratta di un principio euristico e di un dispositivo letterario non dichiarato apertamente dall'autore, con una dissimulazione legata a esigenze di cautela e di schermo.

Una delle novelle per cui la ricerca dei rapporti sistematici tra racconto e dedicatario conduce a esiti di particolare evidenza, tali da giustificare l'applicazione di questo procedimento critico anche al resto dell'opera, è la quarta della prima parte, dedicata al segretario Antonello Petrucci (circa I420-I487). Proprio in virtù della priorità logica del dato biografico nel processo inventivo, per illuminare il rispecchiamento deformante cui ho accennato risulta utile tracciare un sintetico profilo di questo personaggio storico. Antonello Petrucci è una figura dal destino tragico, la cui conturbante parabola di fulgida ascesa e rovinosa caduta sembra riflettere, quasi emblematicamente, la storia della dinastia aragonese nel Regno di Napoli. Per il suo fascino e la sua esemplarità la vita di Antonello Petrucci è stata narrata dopo il 1509 da un contemporaneo, l'accademico pontaniano Tristano Caracciolo (circa I437-I522), ${ }^{3}$ che nel suo De varietate fortunae l'ha raccontata come una delle prove più evidenti della crudele imprevedibilità del destino. Il racconto di Caracciolo fu poi ripreso nella Congiura dei baroni composta da Camillo Porzio all'inizio degli anni Sessanta del Cinquecento. ${ }^{4}$

Uno degli aspetti determinanti della vicenda biografica di Antonello Petrucci è da ricercare nei suoi natali umilissimi. Secondo Caracciolo nacque da una famiglia di ortolani: «Constat tamen humillimae fuisse conditionis et fortunae hortulani».5 Il cognome de Petruciis non deve dunque ingannare su una sua possibile estrazione nobiliare. Caracciolo ipotizza che esso possa derivare dai nomi dei genitori, chiamati forse Petruccio e Petruccia. ${ }^{6}$ Il Petrucci fu conosciuto anche come Antonello d'Aversa, con un nome toponimico legato alla sua patria elettiva. Nato a Teano fu infatti allevato ad Aversa. Egli stesso firmerà i suoi primi documenti da segretario con il nome di Antonellus de Aversa. ${ }^{7}$ Non sorprende che Antonello preferisse associare a sé Aversa piuttosto che Teano. Se infatti a Teano era nato come figlio di contadini, ad Aversa era rinato come figlioccio di un notaio. Quando ancora

\footnotetext{
3 Cfr. Mario Santoro, Tristano Caracciolo e la cultura napoletana della Rinascenza, Napoli, Armanni, 1957; Fank Rutger Hausmann, Caracciolo, Tristano, in Dizionario Biografico degli Italiani, Roma, Istituto della Enciclopedia Treccani, 1976, vol. 19, pp. 463-465.

4 Cfr. Elena Valeri, Porzio, Camillo, in Dizionario Biografico, cit., 2016, vol. 85, pp. I36-I40.

5 Tristano Caracciolo, Opuscoli storici editi e inediti, a cura di Giuseppe Paladino, in Raccolta degli storici italiani dal cinquecento al millecinquecento, ordinata da Ludovico Antonio Muratori, tomo XXII, parte I, Bologna, Zanichelli, I934, pp. 7I-I05, la cit. è a p. 97.

6 Cfr. Ibidem.

7 Cfr. Alan Ryder, The Kingdom of Naples Under Alfonso the Magnanimous. The Making of a Modern State, Oxford, Clarendon, 1976, p. 224, nota 36.
} 
vagabondava poverissimo («semique nudulus» lo descrive Caracciolo), ${ }^{8}$ le sue non comuni doti intellettuali furono notate dal vecchio notaio di Aversa Giovanni Ammirato, che lo accolse in casa propria come un figlio. Da questo notaio Antonello ricevette una prima educazione nelle lettere e nei costumi: «literis et moribus». Fu ad Aversa dunque che egli diede prova per la prima volta di quella eccezionale capacità di apprendimento e di quella infaticabilità che, combinate con la sua umile origine, lo condurranno a essere protagonista di una ascesa sociale straordinaria. Nella compresenza del cognome latineggiante "de Petruciis" con l'indicazione di provenienza "de Aversa" è iscritta emblematicamente tutta l'ambiguità inquieta di questo personaggio.

Presto dopo Aversa per Antonello ci fu la capitale del Regno. Notato il «sagax ingenium» ${ }^{9}$ del giovane, il vecchio Giovanni Ammirato decise di affidarlo a Giovanni Olzina, uno dei segretari di Alfonso d'Aragona, che aveva appena conquistato la corona di re di Napoli. Antonello fece così il suo ingresso come giovane di grandi promesse nella cerchia di funzionari-intellettuali legati al Magnanimo. Venne affidato nientemeno che al più grande umanista presente in quel momento a corte: Lorenzo Valla. ${ }^{10} \mathrm{Da}$ quest'ultimo Antonello fu formato perché diventasse atto ad assumere un posto nel collegio di segretari che curavano gli affari di stato più importanti. Non bisogna tuttavia credere che Antonello ricevesse da Lorenzo Valla una formazione da segretario, sufficiente a redigere qualche lettera in un rozzo latino di cancelleria. L'eminente classicista lo fornì di un'istruzione umanistica all'avanguardia, contemplante la padronanza non solo del latino ciceroniano ma anche del greco. In effetti ciò che distingue la biblioteca di Antonello Petrucci da quella degli altri baroni è proprio la presenza cospicua di manoscritti greci. ${ }^{\text {II }}$ Come poi i suoi figli, Antonello fu membro dell'accademia pontaniana.

Non sorprende dunque la gratitudine di Antonello nei confronti del maestro, che a detta di Tristano Caracciolo considerò come una sorta di padre putativo: «perpetuo gratissimus Laurentio Vallae extitit, venerarique ut parentem». ${ }^{12}$ La formazione umanistica ricevuta dal Valla, oltre alle conquiste intellettuali e accademiche, gli consentì di conseguire uffici sempre più importanti come funzionario, conducendolo a diventare in pochi anni l'uomo più potente del Regno dopo il sovrano. Il suo cursus honorum cominciò già

8 Caracciolo, Opuscoli, cit., p. 97; così la cit. successiva.

9 Ibidem, p. 98.

Io Valla stesso era del resto stipendiato come segretario: cfr. Ryder, The Kingdom of Naples, cit., p. 222.

II Cfr. Tammaro De Marinis, La biblioteca napoletana dei re d'Aragona. Supplemento, col concorso di Denise Bloch - Charles Astruc - Jacques Monfrin, in appendice P. José Ruysschaert, Verona, Valdonega, 1947, tomo I, pp. 2I0-2II.

I2 Caracciolo, Opuscoli, cit., p. 98. 
sotto Alfonso il Magnanimo: prima del I458 Antonello ricevette gli uffici di sigillatore e precettore dei diritti del grande sigillo pendente, ${ }^{13}$ oltre a essere eletto guardiano dei porti e misuratore delle vettovaglie. Tuttavia il salto di qualità della sua carriera avvenne dopo la morte di Alfonso I. L'incertezza e la diffidenza instillate dalla prima ribellione dei baroni in occasione della guerra di successione (I459-I464) indussero re Ferrante a riformare radicalmente l'istituto amministrativo della segreteria. Mentre durante il regno di Alfonso le funzioni di segreteria erano svolte da un collegio di più funzionari, Ferrante preferì concentrare tutti gli uffici nelle mani di una sola persona. ${ }^{14}$ La scelta cadde proprio su Antonello Petrucci, probabilmente perché il sovrano riteneva che la sua eccezionale ascesa sociale, favorita dalla monarchia aragonese, rappresentasse una sicura garanzia di fedeltà.

Le fonti cinquecentesche sono concordi nel testimoniare come a partire da questo momento tra il sovrano e Antonello si instaurasse una sorta di rapporto simbiotico. Questa l'efficace formulazione di Camillo Porzio: «Ferdinando [...] non volendo, come il padre, commettere le cose a più persone, ma ad un solo, elesse sopra ogni altro Antonello, e non solamente lo creò Segretario, ma un altro sè stesso».5 $\mathrm{Il}$ potere di Antonello crebbe in modo vertiginoso: divenne il solo depositario dei segreti del re, quasi l'unico intermediario tra Ferrante e il mondo esterno. La sua promozione sociale fu sancita formalmente nel I459, quando ricevette il cavalierato: da questo momento nelle lettere sarà indicato come eques Antonellus de Petrutiis. ${ }^{16}$ L'abilità e soprattutto la lealtà mostrata in un frangente delicatissimo per Ferrante come quello della guerra di successione, quando fu impegnato in difficili missioni diplomatiche presso i feudatari ribelli, ${ }^{17}$ gli permisero di accumulare molti uffici all'inizio degli anni Sessanta. Ai proventi legati alle mansioni amministrative si aggiungevano le vere e proprie tangenti che Antonello incassava per intercedere presso il sovrano. ${ }^{18}$ Oltre all'allestimento della sua preziosa biblioteca e all'acquisto di un principesco palazzo nella zona più prestigiosa di Napoli, in piazza San Domenico nel seggio di Nido, ${ }^{19}$ le copiose ricchezze accumulate, a partire dalla metà degli anni Sessanta, furono impiegate da Antonello per conseguire titoli feudali: tra l'altro la contea di

I3 Per la descrizione e la storia di queste funzioni amministrative cfr. Ryder, The Kingdom of Naples, cit., pp. 218-258.

I4 Cfr. Giuliana Vitale, Sul segretario regio al servizio degli Aragonesi di Napoli, in «Studi Storici», XLIX, 2, pp. 293-32I, in particolare pp. 298-299.

I5 Camillo Porzio, La congiura de' baroni del Regno di Napoli contra il re Ferdinando I, Firenze, Poligrafia italiana, I848, p. Io.

I6 Cfr. De Marinis, La biblioteca napoletana, cit., p. 2II.

I7 Cfr. Alessio Russo, Petrucci, Antonello, in Dizionario Biografico, cit., 2015, vol. 82, p. 770, con rinvio a http://www.treccani.it.

I8 Cfr. ibidem.

I9 Cfr. Vitale, Sul segretario regio, cit., pp. 302-303. 
Policastro nel I465, la contea di Carinola e la baronia di Cucco Marzano, Marzanello, Parete in Terra d'Otranto nel I480. ${ }^{20}$ Questi acquisti, che significavano l'ingresso nei ranghi del baronaggio del Regno, perfezionarono l'ascesa sociale del figlio di ortolani Antonello Petrucci.

Tanta fortuna e influenza non potevano però non suscitare diffidenze e invidie, da parte - in primo luogo - dell'antica nobiltà di sangue, sospettosa e inquieta per la carriera così luminosa di un popolano. ${ }^{21}$ Lorigine principale della sfortuna di Antonello fu tuttavia l'inimicizia e l'avversione dell'erede al trono Alfonso duca di Calabria, insofferente del forte ascendente esercitato dal Segretario sul padre Ferrante. Forse proprio per paura delle ritorsioni del futuro sovrano, che lo accusava di malversazioni a danno delle casse del Regno, ${ }^{22}$ Antonello Petrucci nel 1485 aderì alla congiura contro re Ferrante. Scoperto, fu arrestato a Castel Nuovo, imprigionato nella stessa fortezza, e, dopo due mesi di interrogatori e torture, l'II maggio I487, decapitato per tradimento «spectante populo». ${ }^{23}$ La splendida parabola sociale di Antonello finiva così tristemente sul patibolo, come quella del Julien Sorel di Stendhal, anche egli giovane di umili natali (era figlio del proprietario di una segheria di Verrières), dotato di non comuni capacità intellettuali e interessi letterari.

Tuttavia Masuccio, morto poco dopo la pubblicazione a stampa della sua raccolta (probabilmente nel I476), non fece in tempo ad assistere all'inizio della fine di Antonello Petrucci. La quarta novella del Novellino fu composta probabilmente quando il Segretario aveva appena raggiunto l'apogeo sociale della sua parabola. Ne è testimonianza innanzitutto l'intestazione: "Al magnifico messere Antonello de Petruciis regio unico fido secretario». ${ }^{24}$ Particolarmente significativi sono gli aggettivi «unico» e «fido»: il primo descrive quasi tecnicamente la situazione creata della riforma della segreteria da parte di Ferrante nel I458; il secondo fa riferimento alla fedeltà mostrata da Antonello durante la guerra di successione terminata nel I464.

L'altezza della posizione del dedicatario è sottolineata ancora più enfaticamente nella dedica. Masuccio, segretario del principe di Salerno Roberto Sanseverino dal I463, si rivolge ad Antonello chiamandolo «magnifico mio maiore» (Novellino IV, 2). Questa formula sagacemente allitterante definisce il rapporto tra il segretario maggiore, quello del re di Napoli, e il segretario minore, quello di uno dei feudatari più importanti del Regno. ${ }^{25}$ Tuttavia la

20 Cfr. ibidem, pp. 3I6-3I7, nota 6I.

2I Ibidem, pp. 3I6-3I7.

22 Cfr. Russo, Petrucci, Antonello, cit.

23 Caracciolo, Opuscoli, cit., p. Ioo.

24 Masuccio Salernitano, Il Novellino (con appendice di prosatori napoletani del '4oo), a cura di Giorgio Petrocchi, Firenze, Sansoni, I957, p. 52. Da qui sono tratte le citazioni dal Novellino, con semplice indicazione del numero del capoverso.

25 Si veda la nota di Settembrini al sintagma «magnifico mio maiore» in Masuccio Salerni- 
superiorità del dedicatario rispetto al dedicante non è affermata sul piano strettamente gerarchico ma su quello delle abilità letterarie. Mostrando una precisa coscienza degli interessi retorici di Antonello, Masuccio definisce quest'ultimo «mare d'ogne retorico stile» (ibidem). Il primo periodo della dedica sembra così formulare una captatio benevolentiae quasi umiliante per il dedicante: al cospetto dell'eloquentissimo Antonello lo stile di Masuccio, anche se fosse ispirato da Orfeo o da Mercurio, apparirebbe come il canto stonato di un vecchio mendico al popolo ignorante: «Esistimo, magnifico mio maiore, che volendo dar principio a scrivere a te, mare d'ogne retorico stile, se in me fusse la lira de Orfeo o la eloquenzia de Mercurio, non altramente che un vil canto d'un cieco al grosso vulgo te parrebbe» (ibidem).

L'apparente magnificazione della dedica assume una portata differente, addirittura antifrastica, se letta alla luce delle allusioni all'esperienza biografica del dedicatario contenute nella novella. ${ }^{26}$ Per favorire una più netta comprensibilità di questo sottile apparato allusivo giova riassumerne brevemente il contenuto. Al tempo del matrimonio della regina Giovanna II con Giacomo di Borbone, quindi intorno al I4I5, giunge a Napoli un frate minore chiamato Ieronimo da Spoleto, che diventa subito famoso grazie alla sua predicazione. Un giorno in un convento di frati domenicani di Aversa a Ieronimo è mostrato il cadavere perfettamente conservato di un antico cavaliere di quella città. L'astuto frate subito pensa di poter sfruttare questo corpo intatto per aumentare il successo della sua predicazione. Corrotti i domenicani responsabili della chiesa, Ieronimo stacca il braccio destro da questo cadavere, deciso a servirsene come reliquia per aumentare i guadagni dei suoi traffici simoniaci. Insieme col compagno Mariano da Saona, fra Ieronimo si imbarca su una nave diretta in Calabria. Una tempesta nei pressi di Capri fa approdare l'imbarcazione su una spiaggia nei pressi di Sorrento. I due compagni decidono allora di organizzare a Sorrento l'imbroglio che avevano progettato di realizzare in Calabria.

La mattina seguente allo sbarco, dopo aver ottenuto dall'arcivescovo di Sorrento la convocazione di una grande folla di popolo, fra Ieronimo pronuncia una pomposa orazione, in cui presenta il braccio del cavaliere di Aversa come la reliquia di san Luca evangelista, promettendo la remissione dei peccati a chiunque faccia un'offerta in denaro. Subito dopo l'ostensione

tano, Il novellino nell'edizione di Luigi Settembrini, a cura di Salvatore S. Nigro, Milano, Rizzoli, 20I0, p. I49.

26 L'equivocità del primo periodo della dedica è legata alla possibilità di riconoscere nel «vil canto d'un cieco» cui Masuccio paragona la sua scrittura (apparentemente sminuendola a cospetto delle abilità retoriche di Antonello) un riferimento dissimulato al più grande dei poeti: Omero. L'allusione al poeta greco giocherebbe abilmente con le competenze di grecista del dedicatario, rivendicando sottilmente una superiorità letteraria di Masuccio addirittura nell'ambito specialistico di Antonello Petrucci. Si tratta di una interpretazione che mi riprometto di approfondire in un'altra sede. 
della falsa reliquia irrompe in chiesa fra Mariano mascherato da domenicano, che denuncia ad arte il raggiro di fra Ieronimo protestando la falsità della reliquia. Fra Ieronimo si inginocchia davanti al crocefisso dell'altare maggiore e chiede a Cristo il miracolo di provocare la morte del frate contestatore, che, finita la preghiera del compagno, stramazza incontanente al suolo. Il miracolo, acclamato dalla folla, è raddoppiato da fra Ieronimo, che prega di nuovo Dio chiedendogli di resuscitare misericordiosamente il miscredente. La finta resurrezione di fra Mariano persuade irrevocabilmente della santità del braccio gli ingenui popolani di Sorrento, che accorrono numerosi a venerare la reliquia donando denaro e pietre preziose. Dopo aver imperversato anche in Calabria con questa frode, fra Ieronimo torna a Spoleto, dove, con le ricchezze accumulate grazie alla falsa reliquia, acquista simoniacamente da un cardinale la dignità vescovile.

Mi sembra chiaro come la struttura narrativa della novella entri in risonanza con il tratto più peculiare della biografia del dedicatario Antonello Petrucci: il suo straordinario avanzamento sociale da figlio di ortolani a titolare di contee e baronie. L'approdo della narrazione è infatti la promozione di Ieronimo da frate mendicante a detentore di un vescovado. Questo passaggio a un grado gerarchico superiore, conseguito illegalmente per simonia, è reso possibile da un imbroglio che prevede a sua volta - quasi concentricamente - una sorta di avanzamento indebito: quello del braccio di un cavaliere di Aversa addirittura a sacra reliquia di san Luca evangelista. Il ricercato parallelismo tra il contenuto del racconto e la vita del dedicatario è tutt'altro che generico, supportato com'è da indizi testuali che paiono inequivocabili. Innanzitutto è impossibile non riconoscere nel cadavere perfettamente conservato del «notivole cavaliero» di Aversa (Novellino Iv, 6) un riferimento cifrato al dedicatario, indicato tra l'altro nei documenti, come si è detto, come eques Antonellus e Antonellus de Aversa. Tanto più che gabellando il braccio del cavaliere aversano per quello di san Luca, fra Ieronimo ricorre a titoli burocratici come 'segretario' e 'cancelliere', piuttosto inusuali per designare un evangelista.

La prima occorrenza di queste strane denominazioni è nell'iniziale allocuzione ai sorrentini: «vi voglio mostrare per augmento de vostra divozione una mirabile reliquia, cioè un braccio con la mano destra intera de quello eccellente e glorioso cancellero del nostro redentore Iesù Cristo messere san Luca evangelista» (23). Lo stesso titolo ricorre nella preghiera innalzata da fra Ieronimo affinché punisca il domenicano contestatore: «s'io dico la verità, che questo sia il vero braccio de messere san Luca, tuo dignissimo cancellero, Signor mio [...] manda la tua sentenzia sopra de lui» (34). Come segretario di Cristo san Luca è invece presentato al momento di esibire al popolo la finta reliquia: «Questa è quella felice e santa mano del fidelissimo secretario del Figliol de Dio!» (28). Si tratta di formule improprie per indicare una persona 
sacra che variano quella di «baron messer santo Antonio», usata tre volte da frate Cipolla nel suo fantasmagorico e truffaldino discorso ai certaldesi. ${ }^{27}$ Tuttavia nelle qualifiche di «cancellero» e «secretario», che riducono ironicamente il racconto evangelico della verità cristiana all'opera di un epistolografo di segreteria, non si può non ravvisare una divertita e ammiccante allusione ad Antonello Petrucci, il Segretario per antonomasia, che nel I462 aveva ricevuto tra l'altro l'ufficio della luogotenenza del gran cancelliere e nel I464 quello di portiere della cancelleria. ${ }^{28} \mathrm{~A}$ ciò si può aggiungere che il sintagma "fidelissimo secretario», usato con riferimento a san Luca, pare maliziosamente ricalcare la descrizione di Antonello Petrucci come «fido secretario» nell'intestazione della dedica.

Questo sottile apparato di corrispondenze allusive autorizza a considerare transitivamente valida anche per l'eccezionale carriera di Antonello Petrucci la contestazione di Masuccio contro la beffa del braccio e l'ottenimento simoniaco del vescovado da parte di fra Ieronimo. Da questo punto di vista assume un rilievo particolare l'insistita polemica contro la compravendita di cariche ecclesiastiche, molto accesa ed esplicita nel commento d'autore:

E se presso lo fine de ditta novella ho ditto che 'l nostro fra Ieronimo comperasse il vescovato, e che la simonia abbia cambiato nome, niuno se ne deve maravigliare, atteso che a ciascuno manifesto può essere, che persona alcuna, per vertuosa che sia, [...] possa mai pervenire ad alcun grado di prelatura, si non col favore del maestro de la cecca [id est zecca]; e quella conviensela comprare a l'incanto, como se fa de' cavalli in fiera, ultre le subornazione de' doni e de' pattuiti dinari (48).

Il ricercato rispecchiamento della vita di Antonello Petrucci nella novella di fra Ieronimo mi sembra legittimare il riconoscimento nell'invettiva di Masuccio di una velata e parallela stigmatizzazione della baratteria imputata al Segretario. La compera del vescovado da parte di fra Ieronimo pare adombrare l'acquisto dei titoli feudali da parte di Antonello Petrucci, reso possibile anche dalle tangenti che, come si è detto, gli venivano versate per garantirsi un canale privilegiato con il sovrano. È molto probabile del resto che Masuccio appartenesse alla schiera di coloro che guardavano con diffidenza alla formidabile ascesa sociale di un popolano come Antonello.

27 Decameron VI Io $\$ 9$, II, 44. Il Decameron è citato da Giovanni Boccaccio, Decameron, Introduzione, note e repertorio di Cose (e parole) del mondo di Amedeo Quondam, Testo critico e Nota al testo a cura di Maurizio Fiorilla, Schede introduttive e notizia biografica di Giancarlo Alfano, Milano, BUR, 20I3. La quarta novella del Novellino è costruita attraverso una serie di riprese testuali dalla novella di fra Cipolla, tanto da apparire come una sua originale riscrittura. Conto di fornire in un'altra sede un'analisi particolareggiata di questi rapporti.

28 Cfr. Russo, Petrucci, Antonello, cit. 
Masuccio era fiero esponente della nobiltà cittadina. La prima qualifica che accompagna il nome dell'autore del Novellino nell'intestazione del libro è non a caso l'indicazione della sua condizione aristocratica: «Novellino del nobele materno poeta Masuccio Guardato da Salerno». La polemica sociale anti-contadina è del resto molto esplicita nella dedica della seconda novella ad Alfonso duca di Calabria: «Idio possa presto destruere il purgatorio, a tal che, non possendo de elimosina vivere, andassero a la zappa, unde la maior parte de loro hanno già contratta la origine» (Novellino II, 7). L'insofferenza sociale nei confronti dei rustici è deducibile anche dal dittico di novelle XXXVI e XXXVII. ${ }^{29}$

Possiamo dunque affermare con sufficiente convinzione che, se fosse stato tra la folla adunata l'II maggio I487 di fronte all'arco trionfale del Castel Nuovo per assistere all'esecuzione di Antonello Petrucci, Masuccio non avrebbe potuto trattenere un sorriso di soddisfazione, ammirando la mannaia scesa finalmente sul collo del Segretario corrotto. Nel cadavere dimezzato di colui che era stato per decenni il braccio destro del re Masuccio avrebbe riconosciuto l'effetto di una giustizia intempestiva ma ineluttabile, di una sorta di geometrico contrappasso, che puniva separando in due il corpo di chi in vita aveva unito mostruosamente in sé la natura di contadino e la dignità di feudatario. Nel ghigno compiaciuto di Masuccio non si sarebbe potuto riconoscere alcun segno di compassione e di ammirazione per un uomo eccezionalmente dotato come Antonello Petrucci; sentimenti che invece avrebbero informato il racconto dell'esecuzione composto - ormai a quasi ottant'anni di distanza - da Camillo Porzio:

Era il segretario, per la lunghezza della prigionia, per li tormenti dell'animo e del corpo, cotanto contrafatto, che, conosciuta la virtù dell'uomo, avrebbe indotto a compassione sino a' sassi. Nondimeno, per dimostrare che l'innocenza della vita preterita non gli faceva temere la sopravegnente morte, lietamente il collo sul ceppo adattò; e con miglior fama che fortuna, dipartendosi da questa dolente vita, in due pezzi rimase..$^{30}$

vincenzo.vitale@unibas.ch

29 Cfr. Vitale, La dedica ad Ariete, cit.

30 Porzio, La congiura de' baroni, cit., p. I42. 